\title{
In-hospital Mortality Prediction among Patients with Fractures of Pelvis and Acetabulum in Intensive Care Unit: Machine Learning versus Conventional System
}

\section{Yanrong Cai ( $\nabla$ yanrongcai5@gmail.com )}

University of Heidelberg: Ruprecht Karls Universitat Heidelberg https://orcid.org/0000-0003-0416-4734

Xiang Jiang

Yangzhi Affiliated Rehabilitation Hospital of Tongji University: Shanghai Sunshine Rehabilitation Center

Weifan Dai

Decathlon International

Qinyuan Yu

Yangzhi Affiliated Rehabilitation Hospital of Tongji University: Shanghai Sunshine Rehabilitation Center

\section{Technical advance}

Keywords: Mortality prediction, Pelvic trauma, Acetabular fractures, SAPS II, MIMIC, Machine learning $(\mathrm{ML})$

Posted Date: March 8th, 2021

DOI: https://doi.org/10.21203/rs.3.rs-286007/v1

License: (9) This work is licensed under a Creative Commons Attribution 4.0 International License.

Read Full License 


\section{Abstract}

\section{Background}

Fractures of pelvis and/or Acetabulum are leading risks of death worldwide. However, the capability of inhospital mortality prediction by conventional system is so far limited. Here, we hypothesis that the use of machine learning $(\mathrm{ML})$ algorithms could provide better performance of prediction than the traditional scoring system Simple Acute Physiologic Score (SAPS) II for patients with pelvic and acetabular trauma in intensive care unit (ICU).

\section{Methods}

We developed customized mortality prediction models with $\mathrm{ML}$ techniques based on MIMIC-III, an open access de-defined database consisting of data from more than 25,000 patients who were admitted to the Beth Israel Deaconess Medical Center (BIDMC). 307 patients were enrolled with an ICD-9 diagnosis of pelvic, acetabular or combined pelvic and acetabular fractures and who had an ICU stay more than 72 hours. ML models including decision tree, logistic regression and random forest were established by using the SAPS II features from the first 72 hours after ICU admission and the traditional first-24-hours features were used to build respective control models. We evaluated and made a comparison of each model's performance through the area under the receiver-operating characteristic curve (AUROC). Feature importance method was used to visualize top risk factors for disease mortality.

Results

All the ML models outperformed the traditional scoring system SAPS II (AUROC=0.73), among which the best fitted random forest model had the supreme performance (AUROC of 0.90). With the use of evolution of physiological features over time rather than 24-hours snapshots, all the ML models performed better than respective controls. Age remained the top of feature importance for all classifiers. Age, BUN (minimum value on day 2), and BUN (maximum value on day 3 ) were the top 3 predictor variables in the optimal random forest experiment model. In the best decision tree model, the top 3 risk factors, in decreasing order of contribution, were age, the lowest systolic blood pressure on day 1 and the same value on day 3 .

\section{Conclusion}

The results suggested that mortality modeling with $M L$ techniques could aid in better performance of prediction for models in the context of pelvic and acetabular trauma and potentially support decisionmaking for orthopedics and ICU practitioners.

\section{Background}

The role of big data and artificial intelligence (Al) in medicine has been receiving increasing attention from researchers in both industrial and academic circles. Healthcare giants and interdisciplinary groups 
at universities have combined big data and Al algorithms to build better health profiles and predictive models around individual patients for improved diagnosis and disease treatment. For example, Roche and IBM joined hands in predicting the early risk of diabetes-related chronic kidney disease (CKD) based on real world data; results showed that the Roche/IBM algorithm had the best predictive performance as compared with published ones [1]. FDNA company created a facial image analysis framework called Deep Gestalt by incorporating computer vision and deep learning algorithms to predict genetic syndromes, making enhanced accuracy than reports from clinicians in most of the scenarios [2]. Yet, there remain significant challenges in the convergence of $\mathrm{Al}$ and medicine. Enhanced dialogue and teamwork between two fields need developing towards precision medicine.

Although previous comparison studies suggested that machine learning $(\mathrm{ML})$ methods are superior to traditional regression in terms of real-time risk prediction [3-4], it is the uncertainty that the same method from them can be reproduced to obtain better accuracy for a different patient cohort, not to mention in a distinct clinical context. Thus, efforts to study and implement Al in various biomedical settings are far from sufficient.

In terms of the early mortality rate, fractures of pelvis and acetabulum top the list of orthopedic traumas, for most of which are severe high-energy injuries [5-6]. Many factors make the treatment of pelvic and acetabular fractures challenging and its corresponding high risk of death. First, the fracture site is usually surrounded by extensive soft tissues, which means more bleeding and higher possibility of accompany with large-area trauma when damaged. Second, patients with pelvic and acetabular fractures often suffer from secondary or simultaneous multiple organ injuries, potentially ranging from limbs to thoracic and abdominal organs, urinary system, brain, or and spinal injury [7-8]. Even for a sophisticated doctor with high qualifications, it is full of uncertainty to judge a patient's mortality and the influencing factors through subjective experience.

The development of predicting hospital mortality for ICU patients has roughly gone through three eras since the past 3 decades. The first version of the APACHE (Acute Physiology and Chronic Health Evaluation) scoring model that proposed in 1981 represents the first stage of subjective experience from medical experts. The second stage dates back to the era of statistical analysis with logistic regression, and the third one is the era of machine learning and big data that is in full swing today. Both SAPS and APACHE are death prediction systems that remain the most widely used in clinical practice, which have formed inherent variables and model formulas. However, several external validation studies have suggested that neither the most recent versions of SAPS nor of APACHE succeeded to accurately predict the actual probability of death [9]. Clinicians, therefore, are calling for a data-based auxiliary tool to predict the in-hospital mortality for a better clinical decision-making.

Here, we linked the popular ML methods and the Multiparameter Intelligent Monitoring in Intensive Care III (MIMIC-III) database, with the representative traumatic disease in orthopedics for the first time. To predict mortality among patients with pelvic, acetabular or combined pelvic and acetabular fractures, we established customized ML models including decision tree, logistic regression and random forest by 
capturing two sets of physiological features from Simple Acute Physiologic Score (SAPS) II --- the first set of variables is obtained within 72 hours after ICU admission; the second as control is from first 24 hours. And we compared the ML experiment models with controls and SAPS II to (1) determine whether the model based on the new technique and MIMIC-III can improve mortality prediction under this specific clinical context; (2) to explore whether the use of evolution of physiological features over time, rather than traditional feature snapshots within the first 24 hours, is supportive to produce a better prediction model.

\section{Methods}

Overall, the approval to access to MIMIC III database has been obtained after completing the required online courses and requirements according to the instructional manual on the official homepage (https://mimic.physionet.org/). On the website, two methods were introduced to have access to the dataset: one is to access the metadata on BigQuery, which is a serverless online platform that supports to directly query data using SQL language; the other is to download the database that is composed of comma-separated (CSV) files and import data into the database system on the local server. The latter has more complete functions with all information, supports flexible operation and queries without overloading. Therefore, we first downloaded a virtual machine with the operating system Linux Centos 7, and installed the large-scale relational database PostgreSQL11 (The PostgreSQL Global Development Group, California, USA) on the remote server. Next, we imported the target data with total uncompressed size (6.2G) into Postgres to build the clinical database, utilized SQL scripts to build MIMIC-III database with a collection of materialized views. We finally conducted data processing, feature selection, model building, training and prediction evaluation in Python 3.6.3 (Python Software Foundation, Vienna, Austria).

\section{Dataset and Patients}

The MIMIC III dataset is an openly available database developed by The Laboratory of Computational Physiology at Massachusetts Institute of Technology (MIT), which consists of data from more than 25,000 patients who were admitted to the Beth Israel Deaconess Medical Center (BIDMC) since 2003 and who have been de-identified for information safety [10].

Here, we identified patients who were diagnosed as pelvic, acetabular, or combined pelvic and acetabular fractures according to ICD-9 code and who survived at least 72 hours after the ICU admission. All the data within the first 72 hours following ICU admission were collected and extracted from the MIMIC-III clinical database (version 1.4).

\section{SAPS II and Feature Expansion}

To date, the SAPS II scoring system is known to discriminate potential survivors and non-survivors well for ICU patients and remains the most widely used in clinical practice. Here, the SAPS scores of individual patients were converted to prediction mortality according to the following formula [11]: 
Variables in the SAPS II system only contain physiological parameters (except for variables including age, type of admission and three underlying diseases) within first 24 hours after the ICU admission. However, we hypothesized that models' performance could be further improved by expanding the observation period (from the first 24 hours to 72 hours in ICU) of the physiological predictors. Hence, we here kept the same variables in the original SAPS II scoring system yet captured their values within 72 hours after ICU admission for ML models. Another set of values from the first 24 hours were extracted to establish the control models. The missing values were replaced with the median of each variable.

\section{Establishing Models and Evaluation}

Based on this customized variable selection, logistic regression, decision tree and random forest models were built to predict in-hospital mortality. Here, the candidate samples were randomly divided into two separate subsets, with one taken as the training set and the other as the test set (the assignment proportion is $7: 3$ ). All models were built on a training dataset by using 5 -fold cross-validation -- $80 \%$ of the data were used for training and the remaining $20 \%$ were used for validation -- and the bestperforming models were evaluated on the test set. 16 predictor variables from the first 24 hours (same as SAPS II system parameters) were used as inputs for ML control models, while extended variables from 72 hours were used for the ML experiment models. The outputs were the same as SAPS II model, which were the estimated in-hospital survival probability for enrolled patients. The evaluation procedure was implemented and presented through the cross-validated area under the receiver-operating characteristic curve (AUROC), with graphically illustrated receiver-operating (ROC) curves. The AUROC value could reflect each model's ability to distinguish the prediction target capacity and its overall prediction effect. To visualize the contribution of the predictor variables in ML models, we applied feature importance visualization, which refers to a class of techniques for assigning scores to input features, indicating the relative importance of each feature when making a prediction. Here, a variable importance measure that utilized coefficients for each input variable were used for logistic regression models, while the change in the Gini index was used for decision tree and random forest classifiers [12]. Figure 1 shows the workflow of this study.

\section{Results}

\section{Distribution of Patients}

In total, 313 patients with an ICD-9 diagnosis of fracture of pelvis and/or acetabulum were admitted. After excluding patients with outliers (age>300), we finally enrolled 307 patients (210 males and 97 females), among which 84 expired (marked as 1, 27.4\% mortality) and 223 survived (marked as 0 ) as showed in Figure 2a. Figure 2b shows the admission types of the 307 patients: 28 patients were admitted for unscheduled surgery (9.1\%), with 278 patients for medical reasons (90.6\%) and the left 1 for 
scheduled surgery $(0.3 \%)$. Figure 3 shows the distributions of age and SAPS II score among survivors and non-survivors in the entire patient cohort. Here, we also described the 16 features from first- 24 hours as baseline characteristics of the cohort in Table 1.

Table 1. First-24 hours characteristics of enrolled patients with pelvis and/or acetabulum fractures in MIMIC-III. 


\begin{tabular}{|c|c|c|}
\hline Characteristics & $\begin{array}{l}\text { Survivors } \\
(\mathrm{n}=223)\end{array}$ & $\begin{array}{l}\text { Non-survivors } \\
(n=84)\end{array}$ \\
\hline Age, years, mean (SD) & $49.83(19.96)$ & $66.29(19.69)$ \\
\hline \multicolumn{3}{|l|}{ Sex, n (\%) } \\
\hline Male & $155(69.5 \%)$ & $55(65.5 \%)$ \\
\hline Female & $68(30.5 \%)$ & $29(34.5 \%)$ \\
\hline \multicolumn{3}{|l|}{ Admission type, $\mathrm{n}(\%)$} \\
\hline Medical & $198(88.8 \%)$ & $80(95.2 \%)$ \\
\hline Unscheduled surgical & $24(10.8 \%)$ & $4(0.48 \%)$ \\
\hline Scheduled surgical & $1(0.04 \%)$ & $0(0)$ \\
\hline Heart rate, max, mean (SD) & $114.73(19.54)$ & $119.34(24.29)$ \\
\hline Heart rate, min, mean (SD) & $74.96(14.68)$ & $68.48(15.05)$ \\
\hline Systolic BP, max, mmHg, mean (SD) & $158.86(21.04)$ & $161.43(26.05)$ \\
\hline Systolic BP, min, mmHg, mean (SD) & $93.61(15.07)$ & $85.07(21.37)$ \\
\hline Temperature, $\max ,{ }^{\circ} \mathrm{C}$, mean (SD) & $38.01(0.65)$ & $37.75(1.16)$ \\
\hline Temperature, min, ${ }^{\circ} \mathrm{C}$, mean (SD) & $36.17(0.68)$ & $35.64(1.05)$ \\
\hline GCS, min, mean (SD) & $13.57(2.33)$ & $12.88(3.02)$ \\
\hline 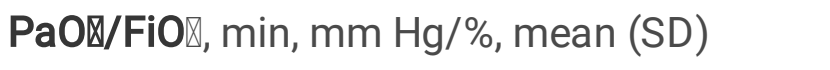 & $248.55(133.16)$ & $195.33(126.77)$ \\
\hline BUN, max, mg/dL or mmol/L, mean (SD) & $20.29(11.66)$ & $29.94(19.97)$ \\
\hline BUN, min, $\mathrm{mg} / \mathrm{dL}$ or $\mathrm{mmol} / \mathrm{L}$, mean (SD) & $12.55(8.26)$ & $20.25(17.58)$ \\
\hline Urine output, mL/day, mean (SD) & $4728.19(2910.69)$ & $4315.20(3334.23)$ \\
\hline Sodium, max, $\mathrm{mEq} / \mathrm{L}$ or $\mathrm{mmol} / \mathrm{L}$, mean (SD) & $141.91(3.60)$ & $143.01(5.62)$ \\
\hline Sodium, min, mEq/L or mmol/L, mean (SD) & $136.07(3.75)$ & $135.95(4.29)$ \\
\hline Potassium, max, mEq/L, mean (SD) & $4.70(0.83)$ & $4.75(0.70)$ \\
\hline Potassium, min, mEq/L, mean (SD) & $3.53(0.40)$ & $3.47(0.47)$ \\
\hline Bicarbonate, max, mEq/L, mean (SD) & $27.93(3.09)$ & $26.40(4.38)$ \\
\hline Bicarbonate, min, $\mathrm{mEq} / \mathrm{L}$, mean (SD) & $22.37(3.89)$ & $21.39(4.46)$ \\
\hline Bilirubin, max, mg/dL, mean (SD) & $1.20(2.08)$ & $1.31(1.43)$ \\
\hline Bilirubin, min, mg/dL, mean (SD) & $0.90(1.47)$ & $0.84(0.68)$ \\
\hline WBC, max, x 103/mm³, mean (SD) & $16.54(7.73)$ & $15.06(6.93)$ \\
\hline
\end{tabular}


Note: Scheduled surgical $=$ surgery scheduled $\geq 24$ hours prior; medical $=$ no surgery within 1 week of admission; unscheduled surgical $=$ surgery scheduled $\leq 24$ hours prior; Abbreviation: $\mathrm{BP}=$ blood pressure; GCS = Glasgow coma scale; $\mathrm{BUN}=$ blood urea nitrogen; $\mathrm{WBC}=$ white blood cells

\section{Comparison among ML Models and SAPS II}

The prediction performance of the optimal logistic regression, random forest and decision tree, and SAPS II scoring is shown in Table 2. Results showed all ML models had better performance of prediction than SAPS II (AUROC $=0.73$, Hosmer-Lemeshow $p<0.001$ ). Among all ML models, the random forest model combined with first-72-hours-SAPS-features achieved the best performance with an AUROC up to 0.90, followed by the decision tree experiment model $(A U R O C=0.89)$ and the logistic regression experiment model (AUROC=0.78).

Table 2. Comparison of customized mortality prediction models with SAPS II.

\begin{tabular}{|llll|}
\hline Models & & AUROC & H-L p Value \\
\hline SAPS II & & 0.73 & $<0.001$ \\
LR & control & 0.76 & 0.37 \\
& experiment & 0.78 & 0.27 \\
DT & control & 0.87 & NA \\
& experiment & 0.89 & NA \\
RF & control & 0.87 & NA \\
& experiment & 0.90 & NA \\
\hline
\end{tabular}

Note: $\mathrm{LR}=$ logistic regression; $\mathrm{DT}=$ decision tree; $\mathrm{RF}=$ random forest; $\mathrm{NA}=$ not applicable

As is shown in Table 2, all the experiment groups with first-72-hours variables performed better than their controls, with comparison of respective ROC presented in Figure 4. For logistic regression models, the Hosmer-Lemeshow $(\mathrm{HL}) \mathrm{p}$ values of both experiment and control models remained non-significant $(>0.05)$, suggesting goodness of fit.

\section{Feature importance}

Top 10 features or risk predictors of each model were showed in Figure 5. Age, BUN (minimum value on day 2), and BUN (maximum value on day 3 ) were the top 3 predictor variables in the best fitted random forest experiment model, while age, BUN (maximum value) and the lowest temperature were top 3 risks of 
mortality in its control models. In the optimal decision tree model, the top 3 risk factors were age, the lowest systolic blood pressure on day 1 and the same value on day3. Although ranking of feature variables varied in different models, age remained the top of feature importance for all classifiers.

\section{Discussion}

The purpose of this study is to establish customized modeling that can provide better performance of mortality prediction for patients with representative orthopedic trauma, as compared to the current standard severity scoring system SAPS II. All models were based on the training data of patients with pelvis and/or acetabulum fractures in the MIMIC-III database. The MIMIC-III database is derived from a large-scale critical care database in the United States. It has a large amount of data, rich variables, and high data quality. Meanwhile, we linked the mainstream ML algorithms with the use of evolution of physiological features over time to further enhance the performance of each model.

A good prediction model should have both satisfying sensitivity and specificity. AUROC, as an index of comprehensive judgment for two were reported here. Based on the results, all the ML models outperformed SPAS II (AUROC of 0.73), among which the random forest topped (AUROC $=0.90$ ) and was followed by the decision tree model (AUROC $=0.89$ ) and then the logistic regression (AUROC $=0.78$ ). The decision tree method is a process of classifying data through a series of rules, which conforms to cosmic human decision thinking. Similarly, the disease diagnosis of a clinician can be regarded as a classification process, that is, the doctor classifies patients into a specific disease group through his knowledge and experience. The results of the decision tree are concise and clear, easy to understand, and helpful to extract the corresponding diagnosis rules. Its application to the classification and diagnosis of diseases can often improve the diagnosis accuracy, making it widely used in clinical practice. In this study, the AUROC of the optimal decision tree model is higher than the traditional SAPS II system ( 0.89 vs $0.73)$, reflecting an improved prediction performance.

Although the decision tree achieves the best balance between accuracy and interpretability, the single decision tree model always fails to achieve the most ideal prediction accuracy. Therefore, scholars have developed a new algorithm based on the decision tree called random forest. As its name suggests, a random forest is composed of multiple decision trees, each of which has undergone relatively independent training and has its own independent prediction and classification capability. On the other hand, if we compare each tree in the model to an individual expert, the random forest model effectively avoids an individual's misjudgment and bias as a result of the combination of multiple experts' voting according to certain rules. In this view, we can obtain a better under-standing of the reason why a random forest performs better than a decision tree. In this study, we obtained the consistence on this point that the best fitted random forest model performs best among all ML models, with an AUROC of 0.90 versus 0.89 in the decision tree. This is also consistent with the results of previous studies. For example, Fernandez-Delgado et al. compared more than 100 model algorithms based on 121 datasets and found that the random forest algorithm has the highest prediction accuracy [13]. Pirracchio et al. established a death prediction model based on the clinical data of 24,508 ICU patients [14]. The results showed that the 
"Super Computing" model customized by combining multiple machine learning algorithms outperformed the disease scoring system, with the finding that the random forest model and the "Super Compu-ting" is comparable in prediction performance (each contained an AUROC as high as 0.880). However, the performance of the model is unavoidable to vary with the application to differing scenarios. Through a single-center clinical study, Mao et al. compared the predictive value of a logistic regression model and two other ML algorithms (decision tree and support vector machine) in early disease warning [15]. The results showed that the predictive accuracy of logistic regression was higher than the other two. Badriyah et al. applied a decision tree model to predict the probability of inpatients in general wards to enter the ICU, and the results showed that its prediction accuracy is comparable to the traditional National Early Warning System (MEWS) [16]. In other fields, logistic regression is more accurate than other machine learning methods. Yet in this study, the AUROC of the logistic regression model with extended variables is 0.78 , which is less than that of the random forest model.

The results revealed an increasing AUROC when comparing each set of ML models before and after feature expansion: the physiological parameters included in SAPS II as key factors were kept, yet we expanded the features by capturing the measurements obtained within the first 72 hours rather than 24hours snapshot after ICU admission. This suggests that the evolution of physiologic variables over time is more predictive to the clinical outcome than the physiologic snapshot of the ICU patients on admission, which is the basis of current severity scoring systems. The result from this improvement of method is consistent with the experience from clinical observation: it is not the initial presentation after ICU admission yet how a patient responds to treatments reflects the trend of deterioration or improvement, thus determining the outcome of each patient. The similar method was used by Celi et al. [17] who reported that the use of the evolution of physiologic variables over time facilitated the ML models' performance in predicting mortality among ICU patients with acute kidney injury (AKI). This method of feature selection may set a good example for the integration of clinician's experience and Al's strength.

Comprehensively, the top 3 important factors affecting the morality of pelvic and/or acetabular fractures were age, systolic BP, and BUN. In our findings, the mean value of age of the non-survivors is 66.29 , which is significantly higher than that of the survivors. Similarly, some studies reported that patients aged greater than 65 with pelvic fractures had higher case fatality despite equivalent measures of injury severity, with a mortality rate of approximately 20 percent [18]. Moreover, both the systolic BP and BUN are indicators of extensive bleeding. According to the results of a retrospective cohort study, a substantial number of patients with pelvic injuries died within the first 24 hours of hospital arrival primarily due to massive hemorrhage [19]. Our finding is consistent with literature [20-21] reporting that bleeding-related risks are reliable risk factors predictive of early mortality, suggesting the significance of initial bleeding control in the management of hemorrhagic shock.

Our study has some limitations. First, the patient cohort was from a single center in the United States, and the number of enrolled patients didn't meet the standard of a set of big data. In addition, this study only conducted internal verification of the model and limited ML methods were compared with each other. In all, further research is needed to verify the external data to generalize the model for other settings. What's 
more, MIMIC-III in this study is not a specialized database for orthopedic trauma, which means there is a lack of relevant detailed information (such as clinical classification of fractures of pelvis and acetabulum, patient trauma scores, etc.) which could be potential features of importance. As ML algorithms and big data are complementary, enhanced dialogue and teamwork between computer scientists, data scientists and clinicians could play a significant role in the progression of $\mathrm{Al}$ in medicine.

\section{Conclusion}

To our knowledge, this study is the first to introduce ML models applied to decision support for patients with the representative traumatic disease in orthopedics. We found that customized modeling with $\mathrm{ML}$ methods could produce better performance of mortality prediction than the traditional severity scoring system SAPS II in the context of pelvic or/and acetabular fractures, potentially facilitating clinical decision-making for orthopedist or ICU practitioners in practice. While evidence-based medicine has overshadowed empirical therapies, we consider that integrated experience with both fields of Al and medicine represents the future of personalized clinical medicine.

\section{Abbreviations}

ML: Machine Learning; SAPS: Simple Acute Physiologic Score; ICU: Intensive Care Unit; BIDMC: Beth Israel Deaconess Medical Center; AUROC: Area Under the Receiver-operating Characteristic Curve; CKD: Diabetes-related Chronic Kidney Disease; APACHE: Acute Physiology and Chronic Health Evaluation; MIMIC: Multiparameter Intelligent Monitoring in Intensive Care; BP: Blood Pressure; GCS: Glasgow Coma Scale; BUN: Blood Urea Nitrogen; WBC: White Blood Cells; LR: Logistic Regression; DT: Decision Tree; RF: Random Forest; HL: Hosmer-Lemeshow

\section{Declarations}

\section{Acknowledgements}

The authors would like to thank the developers who built the MIMIC database and provided the open access for this study.

\section{Competing interests}

The authors declare that they have no competing interests.

\section{Ethics approval and consent to participate}

The use of the MIMIC database was approved by the institutional review boards of both Beth Israel Deaconess Medical Center and Massachusetts Institute of Technology and requirement for individual patient consent was waived according to IRB protocol 2001-P-001699/3 ${ }^{[22-23]}$. 
Not applicable

\section{Funding}

This research did not receive any specific grant from funding agencies in the public, commercial, or notfor-profit sectors.

\section{Data availability}

All the data utilized in our research came from the MIMIC-III database, which can be accessed from https://mimic.physionet.org/ after a permission according to the steps we described in methods section.

\section{Author contributions}

Weifan Dai and Xiang Jiang processed the data, performed the analysis, drafted the manuscript and designed the figures. Qinyuan Yu was involved in the data visualization. Yanrong Cai revised the manuscript, interpreted results and supervised the whole work. All authors have read and agreed to the published version of the manuscript.

\section{References}

1. Ravizza S, Huschto T, Adamov A, et al. Predicting the early risk of chronic kidney disease in patients with diabetes using real-world data. Nat Med. 2019;25(1):57-59.

2. Gurovich Y, Hanani Y, Bar O, et al. Identifying facial phenotypes of genetic disorders using deep learning. Nat Med. 2019;25(1):60-64.

3. Held E, Cape J, Tintle N. Comparing machine learning and logistic regression methods for predicting hypertension using a combination of gene expression and next-generation sequencing data. BMC Proc. 2016;10(S7):34.

4. Singal AG, Mukherjee A, Elmunzer JB, et al. Machine Learning Algorithms Outperform Conventional Regression Models in Predicting Development of Hepatocellular Carcinoma: Am J Gastroenterol. 2013;108(11):1723-1730.

5. Guthrie HC, Owens RW, Bircher MD. Fractures of the pelvis. J Bone Joint Surg Br. 2010;92-B(11):14811488.

6. Hak DJ, Smith WR, Suzuki T. Management of Hemorrhage in Life-threatening Pelvic Fracture: J Am Acad Orthop Surg. 2009;17(7):447-457.

7. Hauschild O, Strohm PC, Culemann U, et al. Mortality in Patients With Pelvic Fractures: Results From the German Pelvic Injury Register: J Trauma Inj Infect Crit Care. 2008;64(2):449-455.

8. Testerman GM, West MR, Hensley S. Full-time orthopedic traumatologists enhance value and increase pelvic fracture caseloads at a rural Level I trauma center. Am Surg. 2013;79(5):549-550.

9. GiViTI, Poole D, Rossi C, et al. Comparison between SAPS II and SAPS 3 in predicting hospital mortality in a cohort of 103 Italian ICUs. Is new always better? Intensive Care Med. 2012;38(8):1280- 
1288.

10. Johnson AEW, Pollard TJ, Shen L, et al. MIMIC-III, a freely accessible critical care database. Sci Data. 2016;3(1):160035.

11. Le Gall JR. A new Simplified Acute Physiology Score (SAPS II) based on a European/North American multicenter study. JAMA J Am Med Assoc. 1993;270(24):2957-2963.

12. Churpek MM, Yuen TC, Winslow C, et al. Multicenter Comparison of Machine Learning Methods and Conventional Regression for Predicting Clinical Deterioration on the Wards: Crit Care Med. 2016;44(2):368-374.

13. Fernandez-Delgado M, Cernadas E, Barro S, Amorim D. Do we Need Hundreds of Classifiers to Solve Real World Classification Problems? :49.

14. Pirracchio R, Petersen ML, Carone M, et al. Mortality prediction in intensive care units with the Super ICU Learner Algorithm (SICULA): a population-based study. Lancet Respir Med. 2015;3(1):42-52.

15. Mao Y, Chen Y, Hackmann G, et al. Medical Data Mining for Early Deterioration Warning in General Hospital Wards. In: 2011 IEEE 11th International Conference on Data Mining Workshops. IEEE; 2011:1042-1049.

16. Badriyah T, Briggs JS, Meredith $P$, et al. Decision-tree early warning score (DTEWS) validates the design of the National Early Warning Score (NEWS). Resuscitation. 2014;85(3):418-423.

17. Celi L, Tang R, Villarroel M, et al. A Clinical Database-Driven Approach to Decision Support: Predicting Mortality Among Patients with Acute Kidney Injury. J Healthc Eng. 2011;2(1):97-110.

18. Dechert TA, Duane TM, Frykberg BP, et al. Elderly Patients with Pelvic Fracture: Interventions and Outcomes. Am Surg. 2009;75(4):291-295.

19. Wang H, Robinson RD, Moore B, et al. Predictors of early versus late mortality in pelvic trauma patients. Scand J Trauma Resusc Emerg Med. 2016;24(1):27.

20. Mardanpour K, Mardanpour N, Elham Zarei MR. Incidence of pelvic trauma and relative risk factors for mortality: a population based study in Iran. Clin Pract. Published online 2018. doi:10.4172/clinical-practice.1000401

21. Papakostidis C, Giannoudis PV. Pelvic ring injuries with haemodynamic instability: efficacy of pelvic packing, a systematic review. Injury. 2009;40:S53-S61.

22. Ghassemi M, Marshall J, Singh N, Stone DJ, Celi LA. Leveraging a Critical Care Database. Chest. 2014;145(4):745-752.

23. Pirracchio R, Petersen ML, Carone M, et al. Mortality prediction in intensive care units with the Super ICU Learner Algorithm (SICULA): a population-based study. Lancet Respir Med. 2015;3(1):42-52.

\section{Figures}






Figure 1

Flowchart of patient selection and study design 
a)

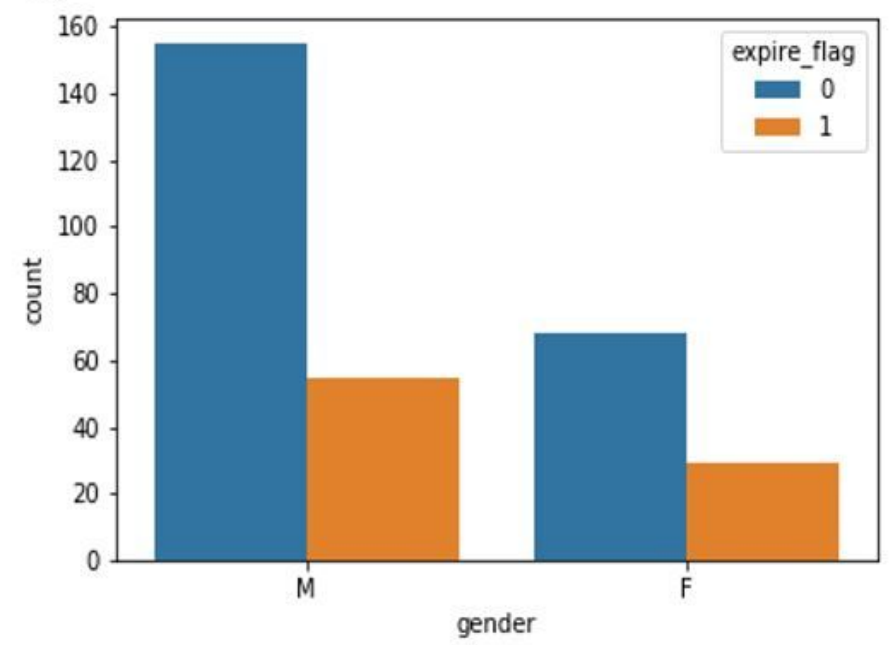

b)

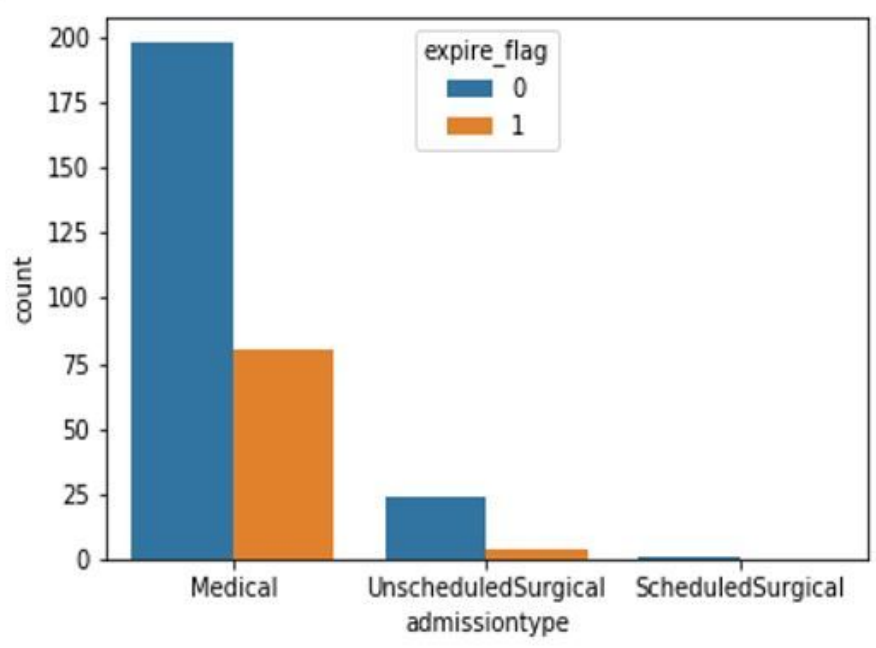

Figure 2

Distributions of the characteristics in the survivors and non-survivors. a) Gender distribution. b) Admission type distribution. Note: Expire flag 0 = survivors; 1 = non-survivors.

a)

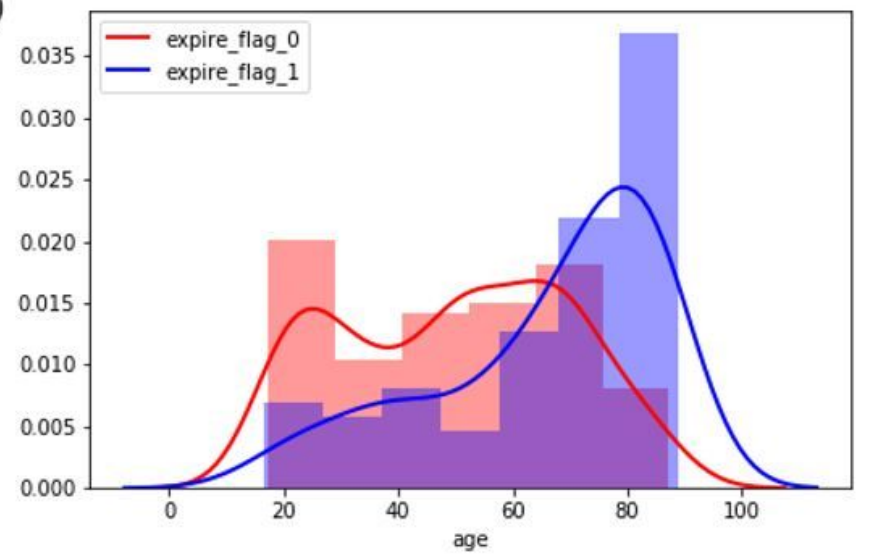

Expire flag $0=$ survivors: $\quad$ Expire flag $1=$ non-survivors:

Mean $=49.627$

$\mathrm{SD}=19.876$
Mean $=66.294$

$\mathrm{SD}=19.691$ b)

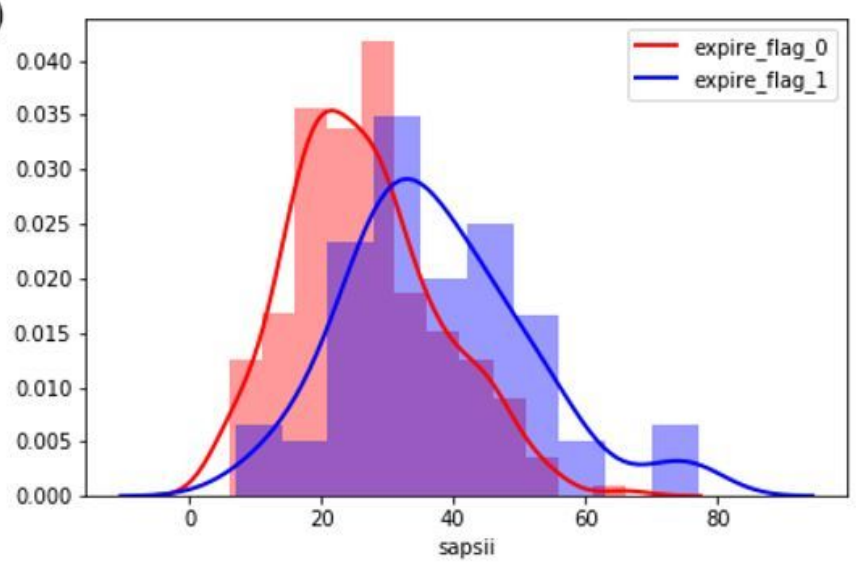

Expire flag $0=$ survivors: $\quad$ Expire flag $1=$ non-survivors:

Mean $=26.471$

$\mathrm{SD}=11.226$
Mean $=37.523$

$\mathrm{SD}=14.177$

\section{Figure 3}

Distributions of selected variables in the survivors and non-survivors. a) Age distribution. b) SAPS II score distribution. Abbreviation: SD = Standard deviation 

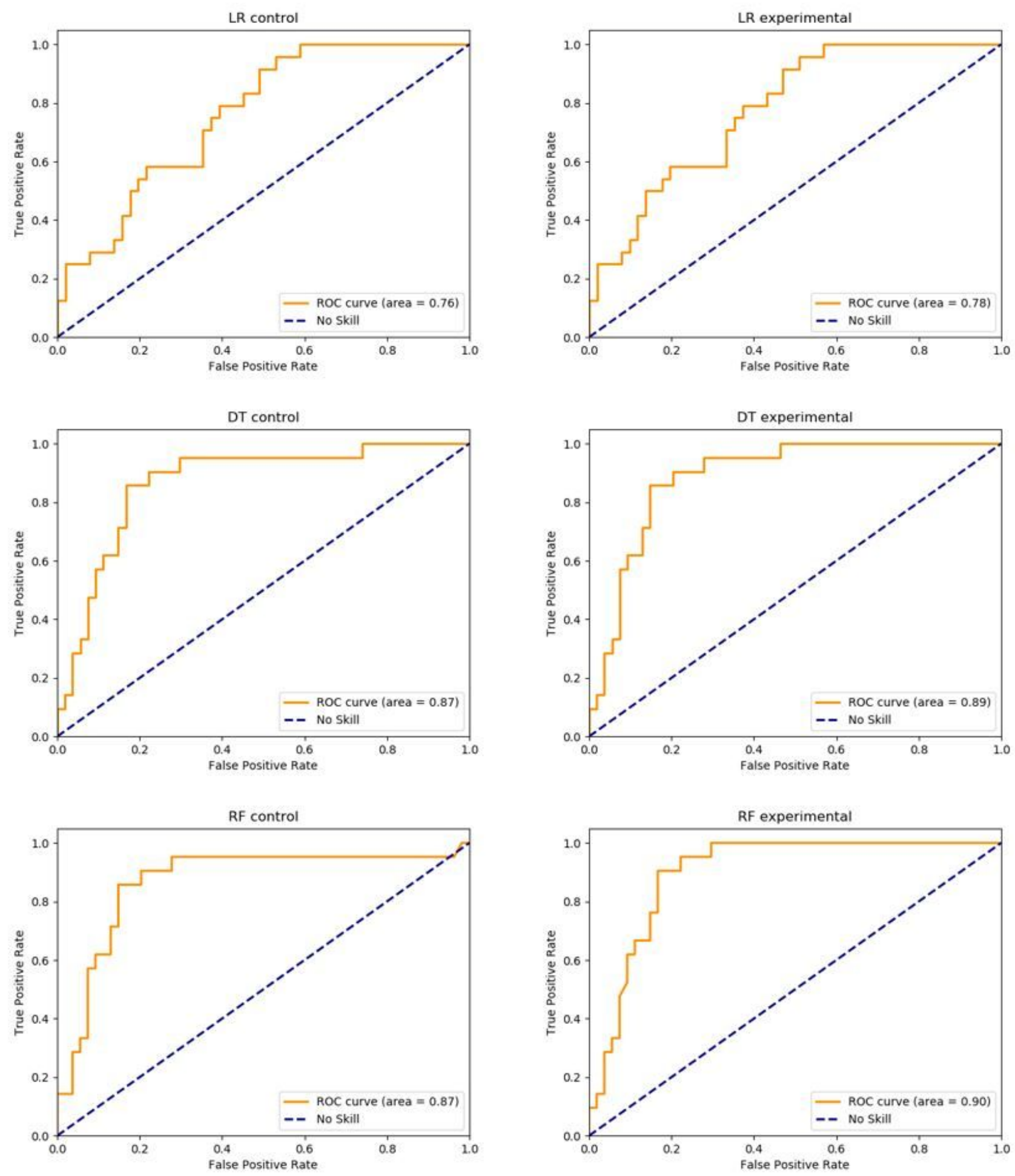

\section{Figure 4}

Receiver-operating characteristics (ROC) curves of customized machine learning models. Abbreviation: $\mathrm{LR}=$ logistic regression; $\mathrm{DT}=$ decision tree; $\mathrm{RF}=$ random forest 

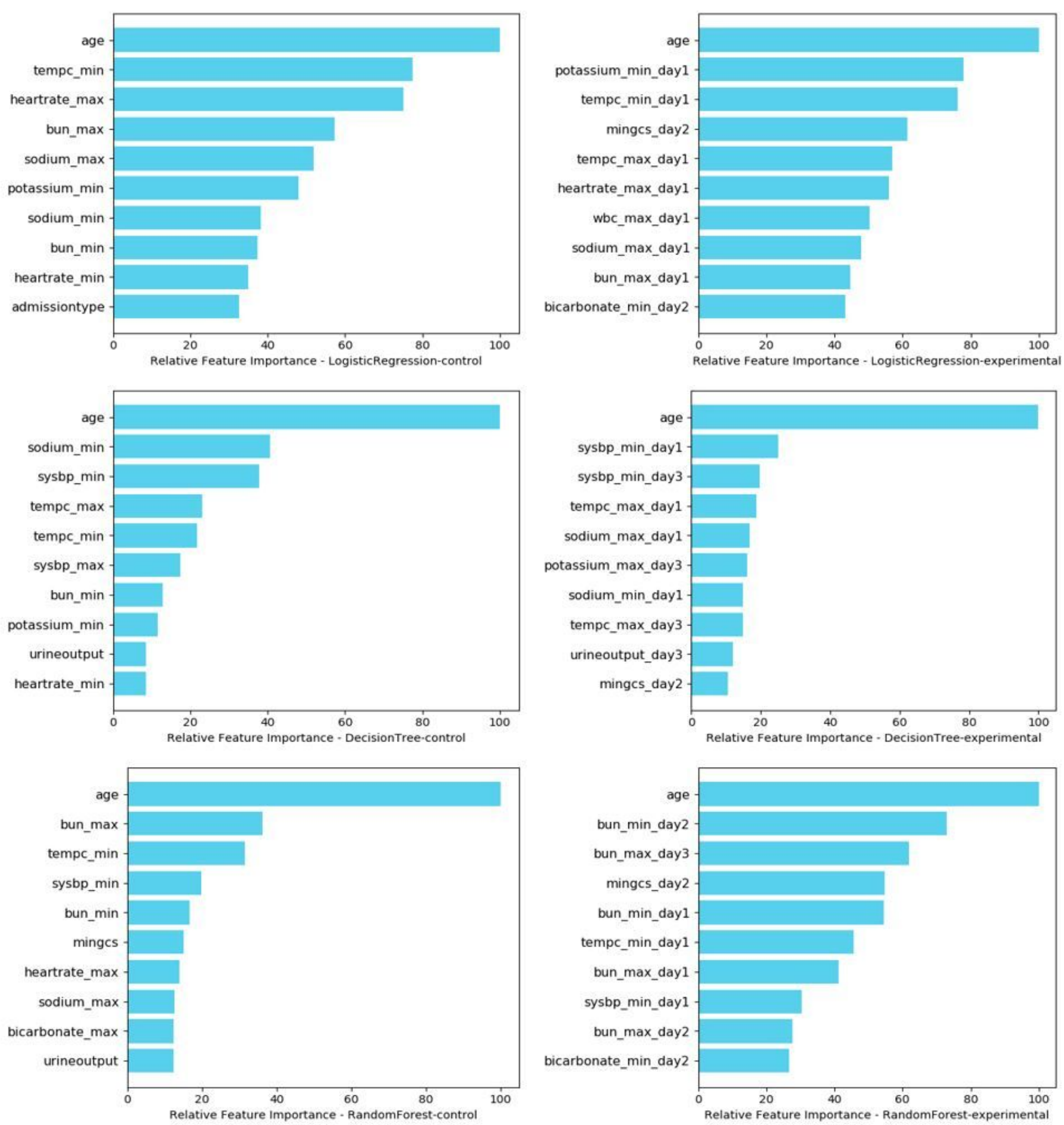

\section{Figure 5}

\section{Feature importance of customized machine learning models.}

